\title{
Magnetic-field-induced binding of few-electron systems in shallow quantum dots
}

\author{
B. Szafran and S. Bednarek \\ Faculty of Physics and Applied Computer Science, AGH University of Science and Technology, PL-30059 Kraków, Poland \\ F. M. Peeters \\ Departement Fysica, Universiteit Antwerpen, Groenenborgerlaan 171, B-2020 Antwerpen, Belgium
}

(Received 24 July 2006; published 15 September 2006)

\begin{abstract}
Binding of few-electron systems in two-dimensional potential cavities in the presence of an external magnetic field is studied with the exact diagonalization approach. We demonstrate that the magnetic field leads to the formation of bound few-electron states which are unbound in the absence of the field. The critical value of the depth of the cavity allowing the formation of a bound state decreases with the magnetic field in a nonsmooth fashion due to the increasing angular momentum of the first bound state. In the high-magnetic-field limit the binding energies and the critical values for the depth of the potential cavity, allowing the formation of a bound system, tend to the classical values.
\end{abstract}

DOI: 10.1103/PhysRevB.74.115310

PACS number(s): 73.21.La, 73.43.-f, 85.35.Be

Quantum dots ${ }^{1}$ (QDs) formed by weak confinement potentials ${ }^{2}$ are susceptible to perturbations by external charge defects and interface roughness. ${ }^{3}$ The spectra of disordered QDs (Refs. 3 and 4) exhibit complex behavior in an external magnetic field, including pairing and bunching of the charging lines, indicating the formation of multiple charged puddles in local potential minima inside the large QD area. In the quantum-Hall effect disorder is held responsible for the formation of localized electron reservoirs that allows for the pinning of the Fermi level between the Landau levels. ${ }^{5}$ Coulomb blockade of charge confined within these tiny reservoirs in quantum-Hall systems have recently been demonstrated ${ }^{6}$ through scanning probe measurements. In this paper we consider electrons localized in a nanometer-size reservoir created by a potential fluctuation or in a shallow quantum dot. The purpose of the present work is to determine the role of the magnetic field in the formation of bound few-electron states.

The present study is related to the problem of the existence of bound excited states of the negative hydrogen ion $\mathrm{H}^{-}$or charged donor center $D^{-}$in a semiconductor. In the absence of a magnetic field the $\mathrm{H}^{-}$has only one bound state, ${ }^{7}$ but an infinite number of weakly bound and arbitrarily extended excited states appears in a weak magnetic field $\left(B \rightarrow 0^{+}\right)$due to the interplay of the magnetic-field-induced localization and the attractive force exerted on the extra electron by the neutral atom $\mathrm{H}$, or donor $D^{0}{ }^{8}$ This force results from the electrostatic potential of $D^{0}$ which is attractive everywhere. ${ }^{8}$ In two-dimensional (2D) quantum wells the $D^{0}$ potential for the extra electron is repulsive at large distances ${ }^{9}$ which prevents the formation of extended excited bound states in the weak magnetic field limit. For off-center $D^{-}$in quantum wells magnetic field induces angular momentum transitions are found, ${ }^{10}$ well known from the theory of QDs. ${ }^{11}$ States with angular momenta $L>4$ cease to be bound which results in magnetic-field-induced $D^{-}$evaporation. ${ }^{10}$

In the symmetric gauge the Hamiltonian of the electron in the magnetic field $(0,0, B)$ contains a diamagnetic term $V_{d}(x, y)=m \omega_{c}^{2}\left(x^{2}+y^{2}\right) / 8$ similar to the two-dimensional harmonic-oscillator potential with the cyclotron frequency $\omega_{c}=e B / m$. Due to the parabolic form of $V_{d}$ one often assumes $^{12}$ that a strong magnetic field overcomes the Coulomb repulsion between the electrons and makes them orbit around the center of mass even in the absence of an external confinement potential. Below we demonstrate that although the magnetic field facilitates the binding of few-electron states, even in the $B \rightarrow \infty$ limit a finite external potential is necessary to stabilize the system. Moreover, we show that the potential parameters stabilizing the system in the $B \rightarrow \infty$ limit can be determined using classical physics.

The electron systems in quantum dots at high magnetic fields are most commonly studied with the parabolic confinement potential. ${ }^{11}$ The parabolic model greatly simplifies the calculations (center-of-mass separation, analytical integration of Coulomb matrix elements, etc.) but it is realistic only in some electrostatic quantum dots where it is a good approximation for the confinement near potential minimum. ${ }^{13}$ However, the price for the numerical simplicity is the limited physics that can be described within the parabolic model. In particular, the parabolic potential possesses only bound states and consequently, it binds an arbitrary number of electrons at any magnetic field. This paper deals with the phenomenon of magnetic-field-assisted binding of few-electron systems in potential cavities. Therefore the application of a potential of a finite size and depth that accounts for the unbound states is a crucial step for the description of the effect. We choose the QD model of a shallow 2D Gaussian well. The Gaussian potential is not only the simplest choice, but also is realized in vertical QDs ${ }^{14}$ with small ${ }^{13}$ gates. The discussed physics is not limited to a Gaussian potential and is independent of the specific choice of the confinement, provided that it has a finite size and depth. A weak magnetic field induces an infinite number of single-electron states weakly bound in the cavity. ${ }^{15}$ On the other hand, the binding of the extended twoelectron states at weak magnetic fields is excluded, since the second electron outside the cavity perceives only the Coulomb repulsion of the electron bound in it. In this paper we look for the effect of the magnetic field that overcomes the Coulomb blockade and allows binding of additional electrons in the cavity. This effect can, for example, be used to 


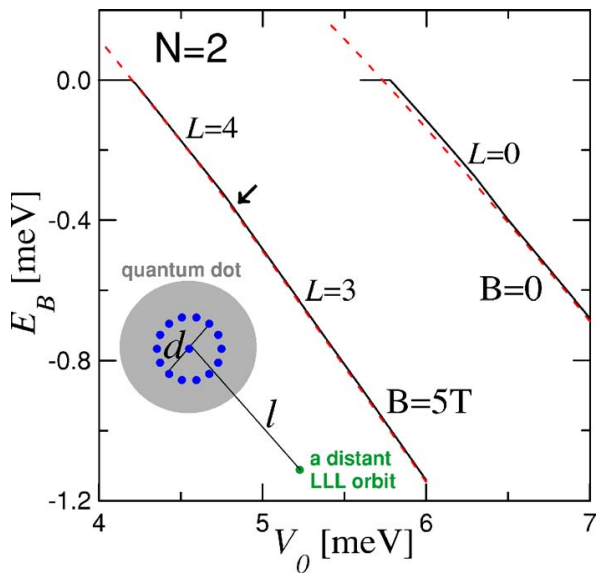

FIG. 1. (Color online) Binding energy of a two-electron system versus potential well depth for $B=0$ and $5 \mathrm{~T}$. Solid lines were calculated using the multicenter basis (the inset shows the choice of centers, $d$ is a variational parameter) and the dashed lines with the finite-difference scheme. The arrow indicates where the groundstate angular-momentum $L$ changes.

entangle spins ${ }^{16}$ of the electrons localized in the cavity and the unbound electrons.

The Hamiltonian of a single electron with the magnetic field $(0,0, B)$ perpendicular to the plane of confinement reads (symmetric gauge),

$$
H=-\hbar^{2} \nabla^{2} / 2 m+V_{d}(x, y)-\frac{1}{2} i \hbar \omega_{c}(x \partial / \partial y-y \partial / \partial x)+V(x, y),
$$

where $m$ stands for the effective band mass (GaAs material parameters are used) and $V(x, y)=-V_{0} \exp \left[-\left(x^{2}+y^{2}\right) / R^{2}\right]$ is the potential well of depth $V_{0}$ and radius $R$ (for the numerical calculations we take $R=50 \mathrm{~nm}$ ). In the absence of the confinement $(V=0)$ the diamagnetic term $V_{d}(x, y)$ induces localization of the single-electron wave functions, but by itself, it cannot keep several electrons together, since the Hamiltonian eigenstates can be localized anywhere. In particular the lowest Landau level (LLL) eigenfunction (with eigenenergy $\left.E_{L L L}=\hbar \omega_{c} / 2\right)$ can be put in the form ${ }^{17}$

$$
\begin{aligned}
\Psi_{L L L}^{(X, Y)}= & (\alpha / 2 \pi)^{1 / 2} \exp \left\{-(\alpha / 4)\left[(x-X)^{2}+(y-Y)^{2}\right]\right. \\
& +i e B / 2 \hbar(x Y-y X)\},
\end{aligned}
$$

where $(X, Y)$ is the arbitrary center of the Landau orbit and $\alpha=e B / \hbar$.

We studied the dissociation of the two-electron confined system using the multicenter configuration interaction approach, ${ }^{18}$ tailored in a way to account for both the bound and unbound states of the two-electron system. The singleelectron states were obtained in the basis of the $\Psi_{L L L}^{(X, Y)}$ wave functions ${ }^{18}$ in which the positions of the centers $(X, Y)$, as well as exponents $\alpha$, are treated as variational parameters. The basis generates also the excited Landau levels and allows for a precise solution of the few-electron Schrödinger equation. ${ }^{18}$ We put 15 centers inside the dot (see the inset to Fig. 1) and a single center localized far away from the dot (at

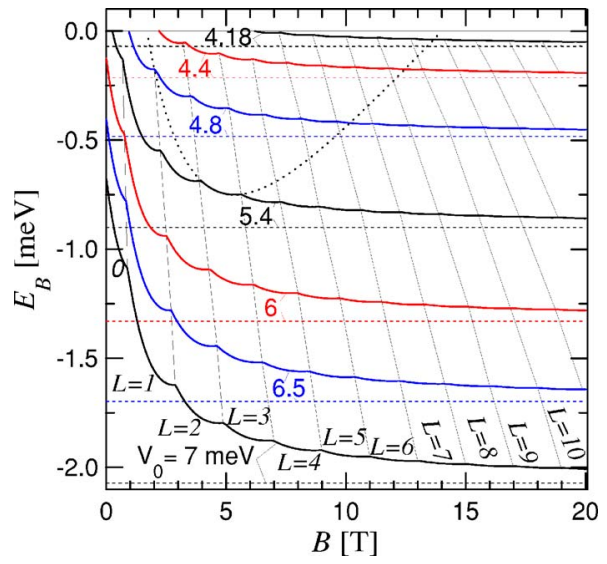

FIG. 2. (Color online) Binding energy of the two-electron system versus the $B$ for depth of the potential from $V_{0}=7 \mathrm{meV}$ (lowest curve) to $4.18 \mathrm{meV}$ (highest curve). Horizontal dashed lines show the binding energies of the classical system. The energy cusps due to the angular-momentum transition are linked by the thin solid lines. The dotted curve shows the binding energy of the state with $L=3$ for $V_{0}=5.4 \mathrm{meV}$.

a distance of $l=2 \mathrm{~cm}$ ). The method ejects an electron from the dot into the LLL localized around the distant center when the two-electron dot-confined system is not bound. Note that the ground state to which the dissociation occurs (with the unbound electron localized on a distant cyclotron orbit center) does not possess the same angular momentum as the initial state. For the unbound $N$ electron states the binding energy

$$
E_{B}(N)=E_{N}-\left(E_{N-1}+E_{L L L}\right)
$$

is not negative. Note that the binding energy is the chemical potential $\mu_{N}=E_{N}-E_{N-1}$ of the $N$-electron system, determining the single-electron charging of the QD devices calculated with respect to the LLL $E_{B}(B)=\mu_{N}-E_{L L L}$.

The calculated binding energies are plotted in Fig. 1 by the thick solid lines as a function of the depth of the well. For $B=0$ the two-electron system becomes bound in the $L=0$ state near $V_{0}=5.75 \mathrm{meV}$. For $5 \mathrm{~T}$ binding appears for a shallower well with $V_{0}=4.2 \mathrm{meV}$, and the first bound state has $L=4$

To verify these results, we used an alternative approach, ${ }^{19}$ using single-electron wave functions diagonalized numerically on a finite-difference mesh. The method ${ }^{19}$ takes into account all the contributing excited Landau levels and allows for an exact description of states localized in a circular potential of arbitrary profile, but does not account for the delocalized LLL's since the calculations are performed within a box of finite size. The results are shown by the dashed lines in Fig. 1 and agree very well with our previous approach. Above the dissociation threshold $E_{B}=0$ the dashed lines become positive due to the absence of the distant LLL in the basis. ${ }^{19}$ The critical $V_{0}$ value is well reproduced. For $B=0 \mathrm{~T}$, the finite difference approach works better than the multicenter basis (see Fig. 1), because it better describes the tails of the wave functions for weakly bound states, which in 


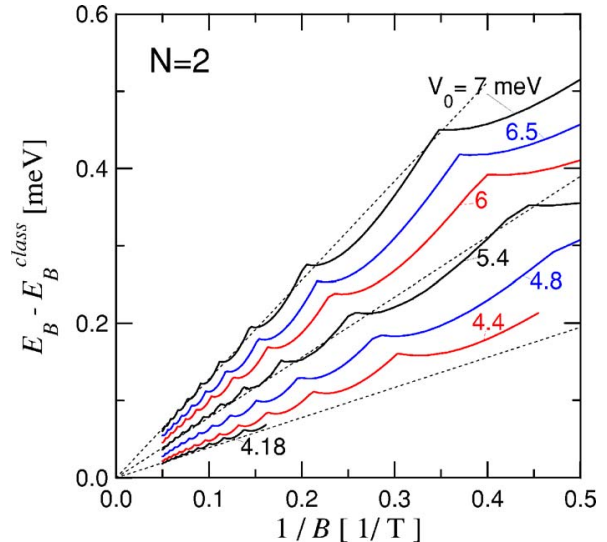

FIG. 3. (Color online) Binding energy of the two-electron system minus the classical binding energy versus the inverse of the magnetic field for depth of the potential from $V_{0}=7 \mathrm{meV}$ (highest curve) to $4.18 \mathrm{meV}$ (lowest curve). The thin dashed straight lines are given by $0.39 / B, 0.78 / B$, and $1.28 / B$.

the absence of a magnetic field can be arbitrarily long. In the following we give the results as obtained with the finite difference method. ${ }^{19}$

The two-electron binding energy as a function of the magnetic field is plotted in Fig. 2 for various depths of the dot. The two electrons are bound for any $B$ when $V_{0}=6 \mathrm{meV}$, or more. The bound ground state undergoes interaction-induced angular-momentum transitions. ${ }^{11}$ The interaction energy increases with the increasing magnetic field untill it reaches a value at which the transition to a more weakly localized state of lower interaction energy is more favorable. At high magnetic fields the angular-momentum transitions ${ }^{11}$ keep the size of the system close to the size of its classical counterpart. ${ }^{20}$ The angular-momentum transitions are associated with the ground-state spin oscillations. States with even $L$ correspond to spin singlets $(S=0)$, and those with odd $L$ to spin triplets $(S=1)$.

For $V_{0}=5.4 \mathrm{meV}$ bound states appear only in the presence of an external magnetic field (see Fig. 2). Notice that, for shallower QDs, the first bound state has nonzero $L$. We found that states of specific $L$ are bound only within a finite interval of the magnetic field (see the binding energy for $L=3$ at $V_{0}=5.4 \mathrm{meV}$ plotted by the dashed line).

The horizonal dashed lines in Fig. 2 show the values of the binding energies obtained for classical point charges, ${ }^{20,21}$ via minimization of the two-electron potential energy $E_{B}^{\text {class }}=\left(e^{2} / 4 \pi \epsilon \epsilon_{0}\right) 1 / w-2 V(w / 2,0)$ with respect to the interelectron distance $w$. The quantum binding energies tend to the classical values at high $B$. This is shown clearly when we plot the difference between the quantum and classical values as function of the inverse of the magnetic field (see Fig. 3). We conclude that the binding energy tend as $1 / B$ to the classical value in the limit of high magnetic fields. ${ }^{22}$ The classical character of the binding is due to the point localization of the single-electron LLL wave functions in the high magnetic field.

The cusps of the three-electron binding energy are due to both the $N=3$ and $N=2$ angular-momentum transitions (cf. Fig. 4). The $1 / B$ convergence of the binding energy to the

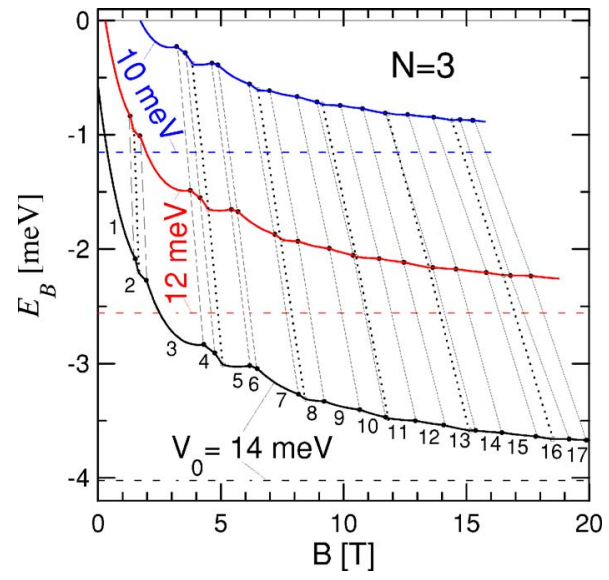

FIG. 4. (Color online) Binding energy of the three-electron system versus the magnetic field for three values of the potential depth. Horizontal dashed lines show the binding energies of the classical system. The energy cusps corresponding to the angular-momentum transitions in the three- two-electron ground state are linked by thin solid (dotted) lines. The numbers below the lowest curve list $L$ for $N=3$.

classical limit is also found for three electrons (see Fig. 5).

Figure 6 shows the "phase diagram" for the maximum number of bound electrons as a function of the well depth and the magnetic field. The thin lines joining the cusps of the phase diagram boundaries corresponding to $N=2$ angularmomentum transitions. The cusps related to the $N=3$ angular-momentum transitions are marked with arrows. Dashed lines show the depth of the potential well which allows binding of the second and third classical electron. ${ }^{21}$ In Fig. 7 we plot the phase diagram as a function of $1 / B$ to demonstrate the convergence of the phase boundaries to the classical result at the infinite magnetic field.

In an external magnetic field with the diamagnetic effect the free-electron LLL increases faster than the energies of the states localized in the cavity. This leads to the energy shift of the ionization threshold observed in a magnetic field. According to our results, the magnetic field induces the formation of bound electron states in QDs. Such a formation was actually observed in single-electron charging experiments on

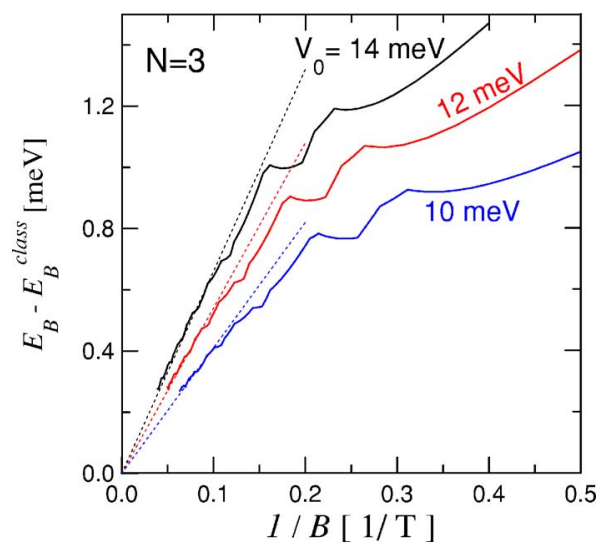

FIG. 5. (Color online) Same as Fig. 3, but for $N=3$. The thin dashed straight lines are given by $6.6 / B, 5.4 / B$, and $4.1 / B$. 


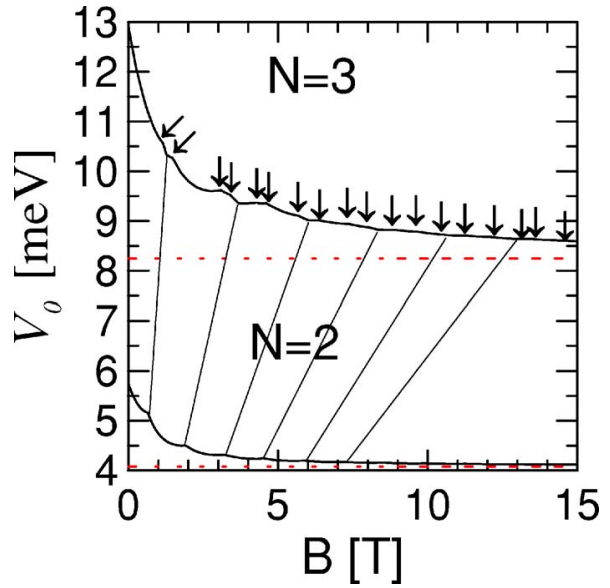

FIG. 6. (Color online) The maximum number of bound electrons on the $V_{0} / B$ plane. Below the lowest curve only one electron can be bound, and above the highest the third electron becomes bound; between the curves the maximum number of bound electrons is two. The horizontal dashed lines show the classical depths of the well, allowing binding of the second and third electron.

self-assembled QDs. ${ }^{23}$ The QDs (Ref. 23) at low magnetic fields bind six electrons. Only for $B>8 \mathrm{~T}$ the capacitance signal related to the binding of the sixth and seventh electrons emerges from the background of the LLL wetting-layer charging (see Fig. 3 of Ref. 23). Self-assembled QDs have a fixed depth of the confinement potential, but the phase diagram (Figs. 6 and 7) can be experimentally determined in the capacitance measurement of the vertical quantum-dot device. ${ }^{14}$ The shape of the confinement potential is fixed by the gate geometry and is exactly Gaussian for a proper choice of the size of the gate (see Fig. 6 of Ref. 13), forming a small electrostatic QD. The depth of the potential is simply changed by the gate voltage. The number of bound electrons can be determined for any magnetic field by counting the capacitance peaks, appearing as a function of the gate volt-

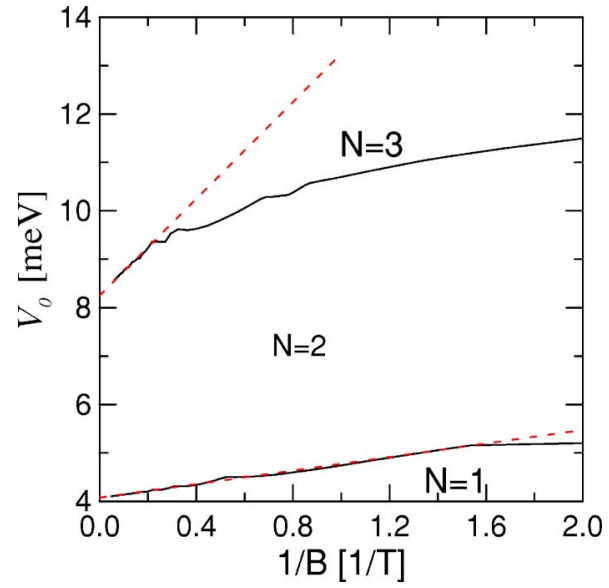

FIG. 7. (Color online) Same as Fig. 6, but in $1 / B$ scale. The thin dashed straight lines are given by $V_{N}^{\text {class }}+A_{N} / B$, with $V_{2}^{\text {class }}=4.073 \mathrm{meV}, V_{3}^{\text {class }}=8.245 \mathrm{meV}, A_{2}=0.7, A_{3}=5$.

age below the 2D electron gas charging (like the one in Fig. 3 of Ref. 23).

The discussed effect can be used to entangle electron spins. ${ }^{16}$ One needs a cavity which at $B=0$ binds only a single electron. The second electron is bound by the field and one can arrange for the bound state to have zero spin (even $L$ ). After the magnetic field is switched off, the second electron will become unbound and is repelled by the dot, and thus goes with its spin state entangled with the localized electron.

In summary, we have shown that a strong magnetic field induces the formation of bound few-electron states in potential cavities that are deep enough to bind the classical point electrons.

This paper was supported by the Foundation for Polish Science (FNP), the Flemish Science Foundation, and by the European Commission network of excellence: SANDiE.
${ }^{1}$ L. Jacak, P. Hawrylak, and A. Wójs, Quantum Dots (SpringerVerlag, Berlin, 1998).

${ }^{2}$ N. B. Zhitenev, R. C. Ashoori, L. N. Pfeiffer, and K. W. West, Phys. Rev. Lett. 79, 2308 (1997).

${ }^{3}$ A. S. Bracker, E. A. Stinaff, D. Gammon, M. E. Ware, J. G. Tischler, D. Park, D. Gershoni, A. V. Filinov, M. Bonitz, F. M. Peeters, and C. Riva, Phys. Rev. B 72, 035332 (2005).

${ }^{4}$ C. M. Canali, Phys. Rev. Lett. 84, 3934 (2000); P. N. Walker, G. Montambaux, and Y. Gefen, Phys. Rev. B 60, 2541 (1999); G. Murthy and H. Mathur, Phys. Rev. Lett. 89, 126804 (2002); B. Reusch and R. Egger, Europhys. Lett. 64, 84 (2003).

${ }^{5}$ S. Datta, Electronic Transport in Mesoscopic Systems (Cambridge University Press, London, 1995); R. B. Laughlin, Phys. Rev. B 23, 5632 (1981).

${ }^{6}$ S. Ilani, J. Martin, E. Teitelbaum, J. H. Smet, D. Mahalu, V. Umansky, and A. Yacoby, Nature (London) 427, 328 (2004).

${ }^{7}$ R. N. Hill, Phys. Rev. Lett. 38, 643 (1977).

${ }^{8}$ J. Avron, I. Herbst, and B. Simon, Phys. Rev. Lett. 39, 1068
(1977).

${ }^{9}$ D. M. Larsen and S. Y. McCann, Phys. Rev. B 45, 3485 (1992); I. K. Marmorkos, V. A. Schweigert, and F. M. Peeters, ibid. 55, 5065 (1997).

${ }^{10}$ C. Riva, V. A. Schweigert, and F. M. Peeters, Phys. Rev. B 57, 15392 (1998).

${ }^{11}$ S. M. Reimann and M. Manninen, Rev. Mod. Phys. 74, 1283 (2002); P. A. Maksym, H. Imamura, G. P. Mallon, H. and Aoki, J. Phys.: Condens. Matter 12, R299 (2000).

${ }^{12}$ R. B. Laughlin, Phys. Rev. B 27, 3383 (1983).

${ }^{13}$ S. Bednarek, B. Szafran, K. Lis, and J. Adamowski, Phys. Rev. B 68, 155333 (2003).

${ }^{14}$ R. C. Ashoori, H. L. Stormer, J. S. Weiner, L. N. Pfeiffer, K. W. Baldwin, and K. W. West, Phys. Rev. Lett. 71, 613 (1993).

${ }^{15}$ The perturbation of the cavity potential is negative for arbitrary LLL angular momentum.

${ }^{16}$ D. P. Di Vincenzo, Phys. Rev. A 51, 1015 (1995).

${ }^{17}$ K. Maki and X. Zotos, Phys. Rev. B 28, 4349 (1983); J. Kainz, S. 
A. Mikhailov, A. Wensauer, and U. Rössler, ibid. 65, 115305 (2002); C. Yannouleas and U. Landman, ibid. 66, 115315 (2002).

${ }^{18}$ B. Szafran, F. M. Peeters, S. Bednarek, and J. Adamowski, Phys. Rev. B 69, 125344 (2004); B. Szafran, F. M. Peeters, and S. Bednarek, ibid. 70, 205318 (2004); B. Szafran and F. M. Peeters, ibid. 71, 245314 (2005).

${ }^{19}$ B. Szafran, F. M. Peeters, and S. Bednarek, Phys. Rev. B 70, 125310 (2004); B. Szafran and F. M. Peeters, ibid. 72, 155316 (2005).

${ }^{20}$ V. M. Bedanov and F. M. Peeters, Phys. Rev. B 49, 2667 (1994).
${ }^{21}$ A. Farias and F. M. Peeters, Solid State Commun. 100, 711 (1996).

${ }^{22}$ The $1 / B$ scale is taken from our previous paper B. Szafran, S. Bednarek, J. Adamowski, M. B. Tavernier, E. Anisimovas, and F. M. Peeters, Eur. Phys. J. D 28, 373 (2004), where it was applied to demonstrate the convergence of the exact and semiclassical HF energies, for Wigner molecules at high $B$.

${ }^{23}$ B. T. Miller, W. Hansen, S. Manus, R. J. Luyken, A. Lorke, J. P. Kotthaus, S. Huant, G. Medeiros-Ribeiro, and P. M. Petroff, Phys. Rev. B 56, 6764 (1997). 